\title{
Increased mortality in the A/A genotype of the SNP rs28372698 of interleukin 32
}

\author{
URBAN ALEHAGEN $^{1}$, LEVAR SHAMOUN ${ }^{2,3}$, JAN INGVAR DIMBERG $^{4}$ and DICK WÅGSÄTER ${ }^{3}$ \\ ${ }^{1}$ Division of Cardiovascular Medicine, Department of Medicine and Health Sciences, Faculty of Health Sciences, \\ Linköping University, SE-581 85 Linköping; ${ }^{2}$ Division of Medical Diagnostics, Department of Laboratory Medicine, \\ Jönköping County, SE-553 05 Jönköping; ${ }^{3}$ Department of Medical Cell Biology, Uppsala University, SE-752 36 Uppsala; \\ ${ }^{4}$ Department of Natural Science and Biomedicine, School of Health and Welfare, Jönköping University, \\ SE-553 18 Jönköping, Sweden
}

Received April 21, 2020; Accepted September 9, 2020

DOI: 10.3892/etm.2020.9559

\begin{abstract}
One of the major causes of mortality in the western hemisphere is cardiovascular disease. Therefore, a variety of markers to identify those at risk are required. Interleukin-32 (IL-32) is a cytokine that is associated with inflammation. The aim of the current study was to investigate variations in single nucleotide polymorphisms (SNPs) of IL-32 and plasma expression, and their associations with mortality. A population of 486 elderly community-living persons were evaluated. The participants were followed for 7.1 years and underwent a clinical examination and blood sampling. SNP analyses of IL-32 rs28372698 using allelic discrimination and plasma measurement of IL-32, using ELISA, were performed. During the follow-up period, 140 (28.8\%) all-cause and $87(17.9 \%)$ cardiovascular deaths were registered. No significant difference between mortality and plasma concentration of IL-32 was observed. The A/A genotype group exhibited significantly higher all-cause mortality $(\mathrm{P}=0.036)$, and an almost two-fold increased risk in a multivariate Cox regression model for all-cause and cardiovascular mortality. A highly significant difference in all-cause and cardiovascular mortality between the $\mathrm{A} / \mathrm{A}$ and the $\mathrm{T} / \mathrm{T}$ groups was demonstrated $(\mathrm{P}=0.015$ resp. $\mathrm{P}=0.014$ ). In the present study, the cytokine IL-32 was demonstrated to have prognostic information, with an increased risk
\end{abstract}

Correspondence to: Professor Urban Alehagen, Division of Cardiovascular Medicine, Department of Medicine and Health Sciences, Faculty of Health Sciences, Linköping University, SE-581 85 Linköping, Sweden

E-mail: urban.alehagen@liu.se

Abbreviations: ACE, angiotensin converting enzyme; CV, cardiovascular; ECG, electrocardiogram; ELISA, enzyme linked immunosorbent assay; HT, hypertension; IHD, ischemic heart disease; IL-32, interleukin 32; SLE, systemic lupus erythematosus; SNP, single nucleotide polymorphisms

Key words: interleukin-32, genotypes, mortality of all-cause and cardiovascular mortality for those with the A/A genotype rs28372698 of IL-32. The A/A genotype could therefore be regarded as a possible biomarker for mortality risk that may be used to offer optimized cardiovascular patient handling in the future. However, the present study sample was small, and the results should be regarded as hypothesis-generating.

\section{Introduction}

Interleukin 32 (IL-32) is an intracellular pluripotent cytokine produced by epithelial cells, monocytes, T lymphocytes and natural killer cells, and is involved in inflammation and cancer development (1). The gene encoding IL-32 is located on the chromosome 16p13.3, organized into eight exons, and it consists of nine splice variants (1). Many inflammatory diseases such as rheumatoid arthritis (2), acute lung injury (3), HIV infection (4), tuberculosis (5), and inflammatory bowel disease (1), are believed to be associated with IL-32 .

An association between IL-32 promoter SNP rs28372698 (A/T), giving a higher IL-32 $\gamma$ gene expression, and thyroid carcinoma has been reported (6). Other studies have demonstrated that this SNP is associated with an increased risk of gastric cancer (7) and endometrial cancer (8). The SNP rs28372698 has also been associated with susceptibility to systemic lupus erythematosus (9) and lung cancer (10). Damen et al (11) reported a possible protective role against cardiovascular disease by the variant rs4786370 in the gene of IL-32. Thus, previous reports indicate that polymorphisms in the gene of IL-32 are important in disease development.

Other studies have focused on levels of serum or plasma of IL-32 between patients and healthy controls. Elevated levels of serum IL-32 in patients with rheumatoid arthritis (2), tuberculosis (5), gastric cancer (12) and heart failure after myocardial infarction (13) have been reported.

The aim of the present study was to explore possible associations between IL-32 SNP rs28372698 and the plasma levels of IL-32 in an elderly group of community-living persons in the south-east of Sweden who were all part of a longitudinal epidemiological study focusing on cardiovascular risk factors with a follow-up period of more than seven years. 


\section{Materials and methods}

Patient population. An elderly population consisting of 486 individuals (males, 247; females, 239) with a mean age of 77.0 years (range, 18 years) living in a municipality in the south-east of Sweden were included in this study. They had all been part of a longitudinal epidemiological study focusing on cardiovascular risk factors (14). The participants in that study were invited to participate in the present sub study conducted from 2003 through 2005. All those living in the municipality within a specific age interval were invited to participate in the longitudinal project in order to minimize bias in the selection process. The population that agreed to participate delivered blood samples, and underwent echocardiographic examinations and an electrocardiogram (ECG). The New York Heart Association functional class [NYHA Class-a functional evaluation where no limitation of activity equates to class $\mathrm{I}$, and symptoms at rest are rated as class IV (15)] was determined by the including physician based on the patient information. The mortality information was obtained from autopsy reports or from the National Board of Health and Welfare in Sweden, which registers all deaths. All participants gave their written informed consent, and the study was conducted in accordance with the principles of the Declaration of Helsinki. The study protocol was approved by the Regional Ethical Review Board of Linköping, Sweden (Dnr 95044).

Co-morbidity. The following definitions have been used in this study. Hypertension (HT) was defined as a blood pressure of more than 140/90 mm Hg measured in the right arm with the patient in the supine position after at least $30 \mathrm{~min}$ of rest. Hypertension was also assumed if the participant had previously been diagnosed with hypertension and was receiving antihypertensive medication. IHD was defined as a history of angina pectoris/myocardial infarction or ECG-verified myocardial infarction. Heart failure was defined as a previous diagnosis with on-going treatment, or symptoms/signs of heart failure and objective demonstration of impaired cardiac function on echocardiography. Cardiovascular death was defined as death caused by fatal arrhythmias, myocardial infarction, heart failure, or cerebrovascular insult. Diabetes mellitus was defined as a previous diagnosis with on-going treatment, or a fasting blood glucose $\geq 7 \mathrm{mmol} / 1$ measured on a single occasion.

Echocardiographic examinations. Echocardiography examinations were performed using an Accuson XP-128c ultrasound system with the patient in a supine, left position. Values for systolic function expressed as left ventricular ejection fraction (EF), were categorized into four classes, with interclass limits of 30,40 and $50 \%$. Normal systolic function was defined as $\mathrm{EF} \geq 50 \%$. Thus, only the systolic function was evaluated.

Determination of IL-32 expression in plasma. All blood samples were obtained at the start of the study, while the patients were at rest in a supine position, and all samples were collected in pre-chilled plastic Vacutainer tubes (Terumo EDTA K-3). Plasma was prepared by centrifugation at $3,000 \mathrm{x}$ g for $10 \mathrm{~min}$ at $4^{\circ} \mathrm{C}$. All samples were stored at $-70^{\circ} \mathrm{C}$ until analysis. None of the samples were thawed more than twice.
Plasma IL-32 levels were measured using a commercially available enzyme-linked immunosorbent assay (ELISA) kit (R\&D Systems) following the manufacturer's protocol. According to the product manual, the kit recognizes human IL-32 $\alpha$, IL-32 $\beta$ and IL-32 $\gamma$. The plasma IL-32 protein concentration from the patients and control subjects was expressed as $\mathrm{pg} / \mathrm{ml}$ and all measurements including plasma and standard solutions for IL-32 standard curves were performed in duplicate.

Genotype determination. Genomic DNA was isolated from all blood samples using QiaAmp DNA Kit (Qiagen). A TaqMan SNP genotype assay was used for analysis of the IL-32 rs28372698 (ID C-64281225-10) (Applied Biosystems). Ten nanograms of DNA was mixed with TaqMan Genotyping Master Mix (Applied Biosystems) and was amplified using the 7500 Fast Real-Time Polymerase Chain Reaction (PCR) system (Applied Biosystems). Amplification was performed using an initial cycle at $50^{\circ} \mathrm{C}$ for $2 \mathrm{~min}$, followed by one cycle at $95^{\circ} \mathrm{C}$ for $10 \mathrm{~min}$ and finally 40 cycles at $95^{\circ} \mathrm{C}$ for $15 \mathrm{sec}$ and at $60^{\circ} \mathrm{C}$ for $1 \mathrm{~min}$. The manual calling option in the allelic discrimination application ABI PRISM 7500 SDS software, (version 1.3.1; Applied Biosystems) was used to assign the genotypes.

Statistical methods. Descriptive data are presented as percentages or mean and standard deviation (SD). Comparative analyses were performed using a Student's unpaired two-sided t-test, whereas the chi-square test was used for discrete variables. Both univariate and multivariate Cox proportional hazard regression analyses were used to analyze and illustrate the risk of mortality during the follow-up period, where both all-cause mortality and cardiovascular mortality were analyzed. Kaplan-Meier graphs were used to illustrate cardiovascular mortality as a function of follow-up time. Censored patients were those who were still alive at the end of the study period or who had died of causes other than cardiovascular disease. Completed patients comprised those who had died due to cardiovascular disease. In the multivariate multivariable regression models, adjustments were made for the following co-variates: IHD, diabetes, ACE inhibitors/Angiotensin receptor inhibitors, beta blockers, diuretics, $\mathrm{EF}<40 \%$, $\mathrm{Hb}<120 \mathrm{~g} / \mathrm{l}$. The variables were chosen because they are well known to influence the risk of cardiovascular mortality.

In the Cox regressions, the A/A genotype of rs 28372698 SNP was evaluated against the $\mathrm{A} / \mathrm{T}$ and the $\mathrm{T} / \mathrm{T}$ genotypes. A P-value $<0.05$ was considered statistically significant. All data were analyzed using standard software packages (Statistica v. 13.2; Statsoft Inc.).

\section{Results}

Study population. The population evaluated consisted of almost equal numbers of males vs. females (247 vs. 239). Of the 486 participants, 112 (23.0\%) had an IHD, and as a result, 36 (7.4\%) had heart failure. In the population, 107 (22.0\%) were classified as having diabetes, and 386 (79.4\%) had HT. As a result of the diseases, $171(35.2 \%)$ were on treatment with beta blockers and 125 (25.7\%) were on ACE inhibitors or angiotensin II receptor antagonists. Thus, the population was 
Table I. Basal characteristics of the study population divided into genotypes.

\begin{tabular}{|c|c|c|c|c|}
\hline \multirow[b]{2}{*}{ Variables } & \multicolumn{4}{|c|}{ Total population } \\
\hline & $\mathrm{A} / \mathrm{A}$ & $\mathrm{A} / \mathrm{T}$ & $\mathrm{T} / \mathrm{T}$ & P-value \\
\hline $\mathrm{N},(\%$ of the total population) & $61(12.6)$ & $226(46.5)$ & $199(40.9)$ & \\
\hline Age, mean $(\mathrm{SD})$ & $76.7(3.4)$ & $77.4(3.6)$ & $76.8(3.2)$ & - \\
\hline $\mathrm{HT}, \mathrm{n}(\%)$ & $43(70.5)$ & $168(74.3)$ & 157 (78.9) & 0.33 \\
\hline IHD, n (\%) & $13(21.3)$ & $56(24.8)$ & 43 (21.6) & 0.67 \\
\hline Diabetes, n (\%) & $10(16.4)$ & $51(22.6)$ & $46(23.1)$ & 0.52 \\
\hline NYHA III, n (\%) & $14(23.0)$ & $44(19.5)$ & $39(19.6)$ & 0.82 \\
\hline $\mathrm{AF}, \mathrm{n}(\%)$ & $8(13.1)$ & $23(10.2)$ & $18(9.0)$ & 0.65 \\
\hline $\mathrm{EF}<40 \%, \mathrm{n}(\%)$ & $5(8.2)$ & $20(8.8)$ & $11(5.5)$ & 0.41 \\
\hline ACEI/ARB, n (\%) & $15(24.6)$ & $59(36.1)$ & $51(25.6)$ & 0.97 \\
\hline $\mathrm{BB}, \mathrm{n}(\%)$ & $21(34.4)$ & $82(36.3)$ & $68(34.2)$ & 0.89 \\
\hline Diuretics, n (\%) & $16(26.2)$ & $93(41.2)$ & $66(33.2)$ & 0.07 \\
\hline $\mathrm{Hb}<120 \mathrm{~g} / 1, \mathrm{n}(\%)$ & 12 (19.7) & $21(9.3)$ & $21(10.6)$ & 0.07 \\
\hline
\end{tabular}

ACEI, ACE-inhibitors; AF, atrial fibrillation; ARB, angiotensin receptor blockers; BB, beta blockers; EF, ejection fraction according to echocardiography; HT, hypertension; IHD, ischemic heart disease; SD, standard deviation; NYHA, New York Heart Association functional class (a functional evaluation where no limitation of activity equates to class I, and symptoms at rest are rated as class IV).

Table II. Distribution of clinical variables between 1st and 4th quartile of plasma levels of IL-32.

\begin{tabular}{|c|c|c|c|}
\hline Variables & $\mathrm{Q} 1<549 \mathrm{pg} / \mathrm{ml}$ & $\mathrm{Q} 4>84,633 \mathrm{pg} / \mathrm{ml}$ & P-value \\
\hline Males, n & 63 & 59 & $\mathrm{~N} / \mathrm{A}$ \\
\hline Females, $\mathrm{n}$ & 52 & 56 & $\mathrm{~N} / \mathrm{A}$ \\
\hline Peripherial edema, n (\%) & $3(2.6)$ & $5(4.3)$ & $\mathrm{N} / \mathrm{A}$ \\
\hline Rales, n (\%) & $18(15.7)$ & $17(14.8)$ & 0.85 \\
\hline Atrial fibrillation, n (\%) & $15(13.0)$ & $6(5.2)$ & 0.04 \\
\hline ACEI/ARB, n (\%) & $30(28.1)$ & $29(25.2)$ & 0.88 \\
\hline Beta blockers, n (\%) & $42(36.5)$ & $49(42.6)$ & 0.34 \\
\hline Diuretics, n (\%) & $47(40.9)$ & $38(33.0)$ & 0.22 \\
\hline $\mathrm{EF}<40 \%, \mathrm{n}(\%)$ & $5(4.3)$ & $10(8.7)$ & 0.18 \\
\hline All-cause mortality, n (\%) & $21(18.3)$ & $26(22.6)$ & 0.41 \\
\hline CV-mortality, n (\%) & $13(11.3)$ & $11(9.6)$ & 0.67 \\
\hline
\end{tabular}

ACEI, angiotensin converting enzyme inhibitor; ARB, angiotensin receptor blocker; CV, cardiovascular; EF, ejection fraction; Q1, 1st quartile; Q4, 4th quartile; IL, interleukin.

representative of an elderly Western population. The distribution of clinical variables in the three genotypes is presented in Table I.

IL-32 levels in plasma. The level of IL-32 in plasma has been evaluated according to quartiles. The distribution of several clinical variables in the two extreme quartiles, the 1st and 4th quartiles, is presented in Table II. The table revealed that no skew distribution regarding clinical variables could be seen in the two quartiles, and no significant difference could be noted regarding the clinical variables between them. The median concentration of IL-32 in the total population was $5782 \mathrm{pg} / \mathrm{ml}$ (range, 69-937920). The CV mortality was evaluated in the 1 st vs. the 4 th quartiles' expression of IL-32
(Fig. 1). No difference in CV mortality between the two groups could be found $(\mathrm{Z}=0.50 ; \mathrm{P}=0.61)$. There were no associations between IL-32 SNP variants and plasma IL-32 levels (data not shown).

Mortality and genotypes. The mean follow-up time of the population was 7.1 years, and during that time 140/486 (28.8\%) suffered all-cause mortality, and 87/486 (17.9\%) suffered CV mortality. From the distribution of the three genotypes in the study population, it could be seen that the A/A genotype was about $1 / 4$ of the other two genotypes (A/T and T/T) (Table I). In an examination of whether different risk groups represented in this study population had different distributions of the three genotypes, those with HT, those with IHD and finally those 
Cumulative proportion surviving (Kaplan-Meier)

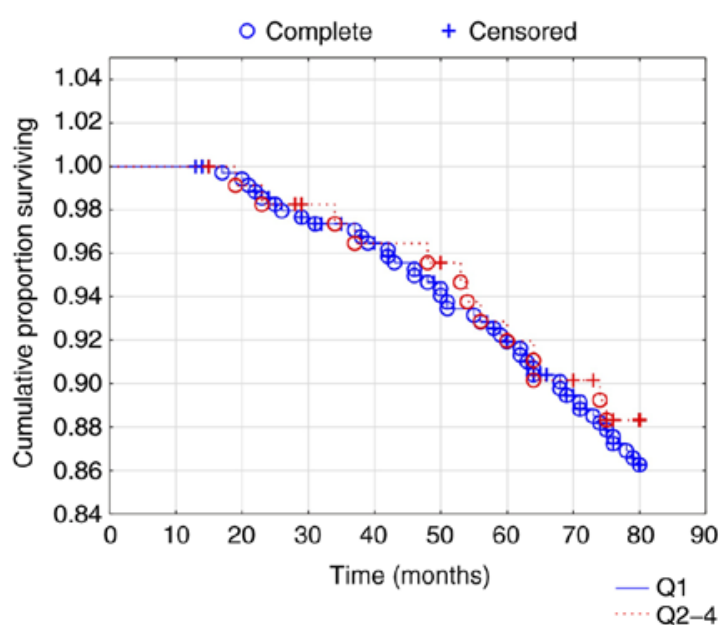

Figure 1. Cardiovascular mortality of those in the first quartile vs. those in the fourth quartile of IL-32 in plasma during a follow-up of 7.1 years. Censored participants were those still living at the end of the study period, or who had died for reasons other than cardiovascular disease. Completed participants were those who had died due to cardiovascular disease. IL, interleukin.

Cumulative proportion surviving (Kaplan-Meier)

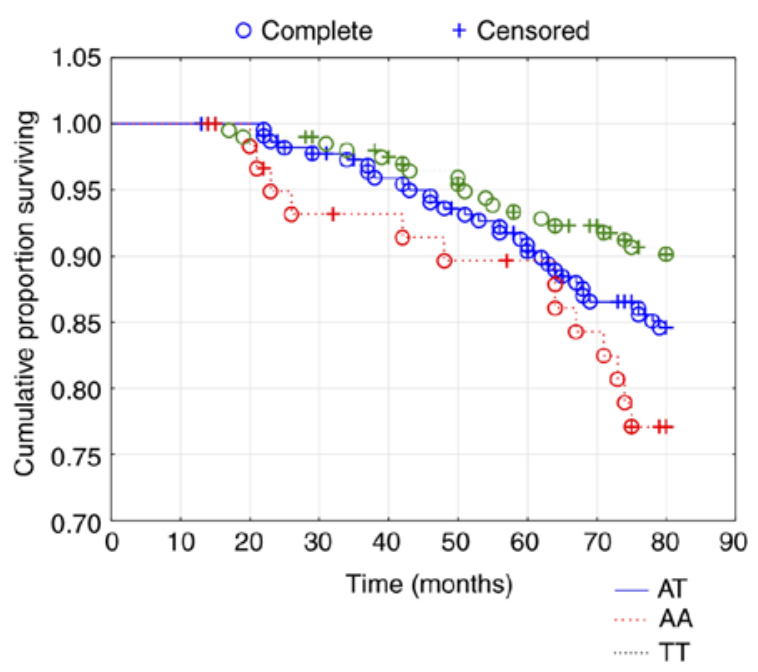

Figure 2. All-cause mortality in the three genotypes of the SNP rs28372698 of IL-32 in the total study population during a follow-up time of 7.1 years. Censored participants were those still living at the end of the study period. Completed participants were those who had died during the follow-up time. SNP, single nucleotide polymorphisms; IL, interleukin.

with diabetes were evaluated. No significant differences in the distribution of the three genotypes compared with the total study population could be seen. Evaluating all-cause mortality in the study population with regard to the three genotypes, a significant difference could be seen where that A/A genotype had the lowest survival regarding all-cause mortality $\left(\chi^{2}: 6.65\right.$; $\mathrm{P}=0.036$ ). In order to validate the result above, a Kaplan-Meier evaluation was performed which showed significant differences $\left(\chi^{2}: 6.51 ; \mathrm{P}=0.039\right)$ between the three genotypes (Fig. 2 ). From the graph it can be seen that the A/A genotype had the lowest survival from CV mortality during the follow-up time of 80 months. Regarding CV mortality, a significantly higher mortality could be found in the A/A genotype compared to

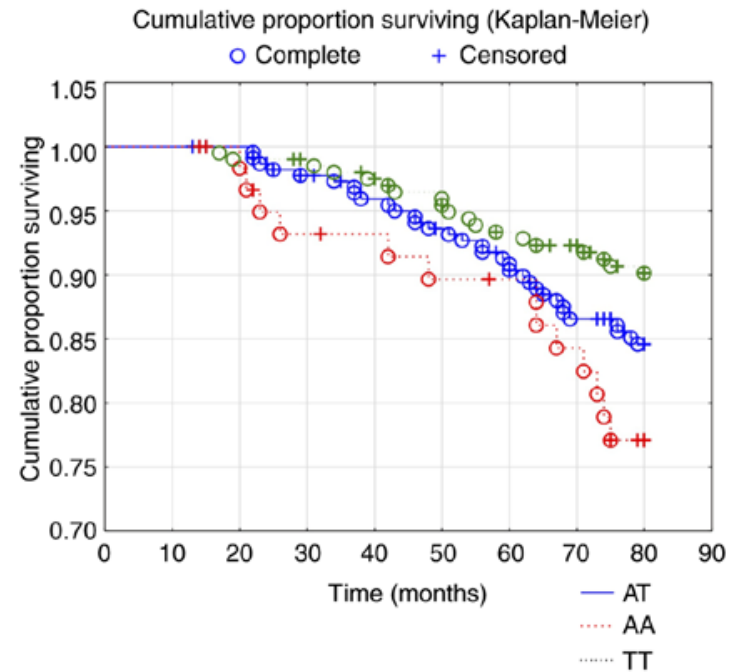

Figure 3. Cardiovascular mortality in the three genotypes of the SNP rs28372698 of IL-32 in the total study population during a follow-up time of 7.1 years. Censored participants were those still living at the end of the study period, or who had died for reasons other than cardiovascular disease. Completed participants were those who had died due to cardiovascular disease. SNP, single nucleotide polymorphisms; IL, interleukin.

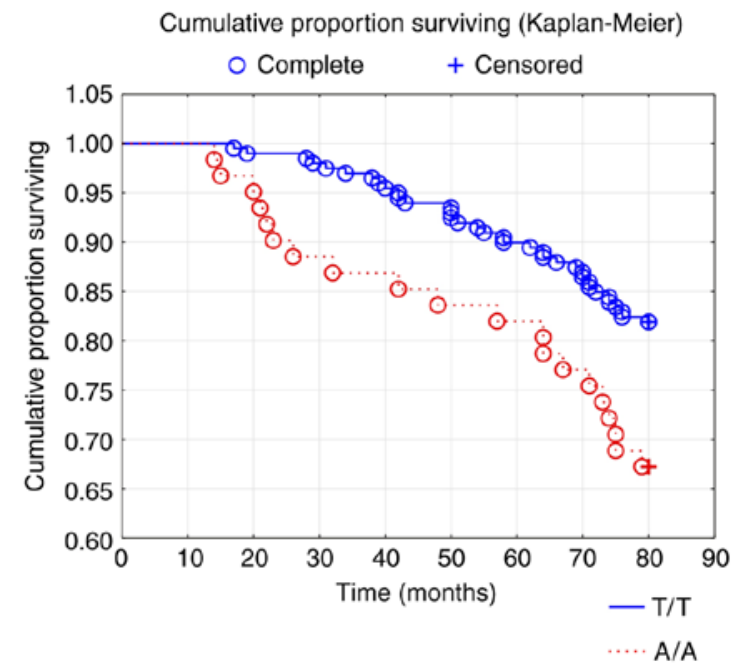

Figure 4. All-cause mortality in the two genotypes A/A vs. T/T of the SNP rs28372698 of IL-32 in the total study population during a follow-up time of 7.1 years. Censored participants were those still living at the end of the study period, or who had died for reasons other than cardiovascular disease. Completed participants were those who had died due to cardiovascular disease. SNP, single nucleotide polymorphisms; IL, interleukin.

the $\mathrm{A} / \mathrm{T}$ and the $\mathrm{T} / \mathrm{T}$ genotypes $\left(\chi^{2}: 5.52 ; \mathrm{P}=0.02\right)$. To validate the obtained results, a Kaplan-Meier analysis was performed, as above, which also showed significantly lower survival from $\mathrm{CV}$ mortality in the A/A genotype, compared to the other two genotypes $\left(\chi^{2}: 6.50 ; P=0.039\right)$, (Fig. 3). Through a second validation a risk evaluation was applied. From that the A/A genotype could be demonstrated to have a 1.8-fold increased risk of all-cause mortality, and an almost two-fold increased risk of CV mortality when including several well-known clinical variables that influence mortality risk (Table III). When analyzing the two genotypes that have the highest difference in mortality, that is $\mathrm{A} / \mathrm{A}$ and the $\mathrm{T} / \mathrm{T}$ genotypes, a significant 
Table III. Cox proportional hazard regression analysis evaluating risk of all-cause- and cardiovascular mortality in the study population regarding rs28372698 of IL-32 during a follow-up period of 7.1 years.

\begin{tabular}{lccccccr}
\hline & \multicolumn{3}{c}{ All-cause mortality } & & \multicolumn{3}{c}{ Cardiovascular mortality } \\
\cline { 2 - 3 } Variables & HR & $95 \%$ CI & P-value & & HR & $95 \%$ CI & P-value \\
\hline IHD & 1.61 & $1.04-2.49$ & 0.03 & & 1.75 & $1.00-3.07$ & 0.05 \\
Diabetes & 1.65 & $1.07-2.53$ & 0.2 & & 1.65 & $0.94-2.87$ & 0.08 \\
ACEI/ARB & 0.77 & $0.49-1.23$ & 0.27 & & 0.81 & $0.44-1.47$ & 0.49 \\
Beta blockers & 0.96 & $0.63-1.46$ & 0.84 & & 0.88 & $0.51-1.54$ & 0.66 \\
Diuretics & 1.21 & $0.80-1.82$ & 0.37 & & 1.07 & $0.62-1.85$ & 0.80 \\
EF <40\% & 2.13 & $1.19-3.80$ & 0.01 & & 2.25 & $1.07-4.76$ & 0.03 \\
Hb <120 g/l & 1.10 & $0.63-1.91$ & 0.74 & & 1.50 & $0.79-2.85$ & 0.22 \\
Rs28372698, IL-32, A/A & 1.84 & $1.13-3.00$ & 0.01 & & 1.96 & $1.06-3.63$ & 0.03 \\
\hline
\end{tabular}

ACEI, angiotensin converting enzyme inhibitors; ARB, angiotensin receptor inhibitors; EF, ejection fraction; HR, hazard ratio; IHD, ischemic heart disease; IL, interleukin; CI, confidence interval.

difference in all-cause mortality $\left(\chi^{2}: 5.97 ; \mathrm{P}=0.015\right)($ Fig. 4$)$, as well as in cardiovascular mortality could be seen $\left(\chi^{2}: 5.99\right.$; $\mathrm{P}=0.014)$.

\section{Discussion}

As health resources are limited, the need for instruments to identify those at high or low risk of cardiovascular complications or mortality is increasing. This report demonstrates an association between the A/A genotype of the IL-32 SNP rs28372698 and increased risk of both all-cause, and cardiovascular mortality in an elderly community-living population, with a follow-up time of 90 months.

In the PubMed database only six reports could be found regarding rs28372698 totally, and just a few regarding genotypes of the SNP and mortality. Wang et al (9) reported an increased susceptibility of SLE in the T/T genotype; however, the role of this polymorphism regarding the function of IL-32 is unknown due to the lack of information in the literature. Regarding information on cardiovascular risks and polymorphisms of IL-32, the situation is the same.

As IL-32 is a cytokine that is involved in inflammatory states, the increased mortality risk might not be surprising. However, this relation between IL-32 and mortality risk has not been reported previously. That there is an intimate association between inflammation and ischemic heart disease is well-known in the literature (16-18). There are also an increasing number of reports indicating that increased oxidative stress is seen when the vascular system is diseased $(19,20)$. Also, endothelial dysfunction, one of the early indicators of cardiovascular injury, is reported to be associated with ischemic heart disease $(21,22)$. The association between IL-32 and cardiovascular risk is even more closely associated with states of chronic inflammation, something that seems logical (23). One of the important factors might be the fact that IL-32 promotes angiogenesis (24). In the literature, there are reports on the genetic polymorphisms of IL-32, and risk. Therefore, as the IL-32 is produced in many of the cell systems involved in inflammation, it is not surprising that this biomarker has an association with disease states that have inflammatory components. There are reports indicating that the plasma concentration of IL-32 is positively associated with prognosis in heart failure after myocardial infarction (13). In our population we could not see a corresponding association, which might be explained by the fact that the population was elderly, and thus had already been exposed to cardiovascular risk factors for a long time, and that the follow-up was proportionally short.

There are reports that have focused on the genetic polymorphisms of IL-32. Shamoun at al. reported no relation to survival for IL-32 SNP rs 28372698 in patients with colorectal cancer (25). Gonzalez-Hormazabal et al (7) reported an interesting interaction between SNP IL-8 rs4073 and SNP IL-32 rs28372698 and a proposed increased risk for gastric cancer for the allele A of rs4073. In our evaluation we were able to present data indicating significant increased mortality, both all-cause, and cardiovascular mortality, in the A/A genotype of rs28372698. However, the size of our study population was limited, so there is uncertainty regarding the result. Because of this, we applied a validation process including two different evaluations (Kaplan-Meier evaluation, and Cox proportional hazard regressions evaluation). Both validations presented the same result, indicating that there might be an increased risk of all-cause and cardiovascular mortality among those with the A/A genotype. However, it is important to stress that the population evaluated were elderly, and thus, those with the highest risk had probably already died, and so the present study population might be characterized as a group of persons with slightly lower risk. That there was still a significant risk for the participants could be seen by the mortality figures during the 90 months of follow-up.

The important question to answer therefore is why the A/A genotype showed a higher risk, as compared to the A/T and the T/T genotypes. This SNP is associated with altered gene expression of IL-32 $\mathrm{g}$ which could affect the inflammatory condition in the patients and also the outcome. It cannot be excluded that the investigated polymorphism may be in linkage disequilibrium with other polymorphisms that 
modulate the susceptibility to the outcome. However, as already mentioned, the lack of reports of rs 28372698 severely restricts the possibility to make comparisons with other reports. Thus, we argue that evaluation of the genotypes of the cytokine IL-32 might give the clinician important knowledge on which of the everyday patients should receive more intense follow-up, with increased prevention measures.

As the results presented are from a community-based study, the majority of patients had discrete or no cardiovascular symptoms. Therefore, those with cardiovascular disease were in a minority and thus the size of the group with cardiovascular disease was small, resulting in wide confidence intervals in risk evaluations, making their interpretation uncertain. However, it could be argued that a message regarding cardiovascular risk was still found. As the message regarding risk of all-cause mortality points in the same direction, it could be interpreted as strengthening the obtained results. The study population was an elderly one, and therefore it was not possible to extrapolate the obtained results into other age groups without uncertainty. As the groups with certain genotypes were small, the results should be interpreted with caution, and should be regarded as hypothesis-generating.

In the present study the SNP rs 28372698 of IL-32 was evaluated regarding all-cause and cardiovascular mortality in an elderly community-living population during a follow-up period of 7.1 years. No difference in mortality could be seen that depended on the plasma concentration of IL-32. However, on evaluating the three genotypes, A/A, A/T and T/T, significantly higher all-cause and cardiovascular mortality could be found in the A/A genotype. This result was replicated in both Kaplan-Meier evaluations and in risk evaluations according to Cox proportional hazard regression analyses. The results are interesting and could give important information in order to better handle patients during a risk stratification at a time with restricted health resources. However, the size of the groups was small and the results should be regarded as hypothesis-generating, so more research is needed.

\section{Acknowledgments}

Not applicable.

\section{Funding}

The present study was supported by grants from the County Council of Östergötland, University of Linköping, Linköping, Sweden, and the Swedish Heart and Lung Foundation.

\section{Availability of data and materials}

The datasets generated and/or analyzed during the current study are not publicly available due to that under Swedish Law, the authors cannot share the data underlying this study and cannot do any further research than what is specified in the ethical permissions application. For inquires on the data, researchers should first reach out to the owner of the database, the University of Linköping. Please reach out to the corresponding author with requests and for assistance with data requests. If the university approves the request, researchers can submit an application to the Regional Ethical
Review Board for the specific research question that the researcher wants to examine.

\section{Authors' contributions}

UA conceived and designed the experiments, performed the experiments, analyzed the data and wrote the manuscript. DW conceived and designed the experiments, performed the experiments, analyzed the data, contributed reagents/material/analysis tools. LS performed the experiments and contributed reagents/material/analysis tools. JID conceived and designed the experiments, performed the experiments, contributed reagents/material/analysis tools and wrote the manuscript.

\section{Ethical approval and consent to participate}

The current study was conducted in accordance with the Declaration of Helsinki principles. The study protocol was approved by the Regional Ethical Review Board of Linköping, Sweden (Dnr 95044).

\section{Patient consent for publication}

All participants gave their written informed consent to participate and to allow data being published, and the.

\section{Competing interests}

The authors declare that they have no competing interests.

\section{References}

1. Hong JT, Son DJ, Lee CK, Yoon DY, Lee DH, Yoon DY, Lee DH and Park MH: Interleukin 32, inflammation and cancer. Pharmacol Ther 174: 127-137, 2017.

2. Gui M, Zhang H, Zhong K, Li Y, Sun J and Wang L: Clinical significance of interleukin-32 expression in patients with rheumatoid arthritis. Asian Pac J Allergy Immunol 31: 73-78, 2013.

3. Arcaroli JJ, Liu N, Yi N and Abraham E: Association between IL-32 genotypes and outcome in infection-associated acute lung injury. Crit Care 15: R138, 2011.

4. El-Far M, Kouassi P, Sylla M, Zhang Y, Fouda A, Fabre T, Goulet JP, van Grevenynghe J, Lee T, Singer J, et al: Proinflammatory isoforms of IL-32 as novel and robust biomarkers for control failure in HIV-infected slow progressors. Sci Rep 6: 22902, 2016.

5. Bao F, Wen X, Liu A, Dai X, Zhao Q, Wang Y and Lu S: Elevated levels of serum IL-32 in patients with active pulmonary tuberculosis. Afr J Microbiol Res 6: 7292-7294, 2012.

6. Plantinga TS, Costantini I, Heinhuis B, Huijbers A, Semango G, Kusters B, Netea MG, Hermus AR, Smit JW, Dinarello CA, et al: A promoter polymorphism in human interleukin-32 modulates its expression and influences the risk and the outcome of epithelial cell-derived thyroid carcinoma. Carcinogenesis 34: 1529-1535, 2013.

7. Gonzalez-Hormazabal P, Musleh M, Bustamante M, Stambuk J, Escandar S, Valladares H, Lanzarini E, Chiong H, Rojas J, Castro VG, et al: Role of cytokine gene polymorphisms in gastric cancer risk in Chile. Anticancer Res 34: 3523-3530, 2014.

8. Yu X, Zhou B, Zhang Z, Gao Q, Wang Y, Song Y, Pu Y, Chen Y, Duan R, Zhang L and Xi M: Significant association between IL-32 gene polymorphisms and susceptibility to endometrial cancer in Chinese Han women. Tumour Biol 36: 5265-5272, 2015.

9. Wang Y, Zhou B, Zhao Y, Yu X, Liu Y and Zhang L: Association of plasma IL-32 levels and gene polymorphisms with systemic lupus erythematosus in Chinese Han population. Dis Markers 2016: 2460206, 2016. 
10. Wang Y, Yang Y,Zhu Y,Li L, Chen F and Zhang L: Polymorphisms and expression of IL-32: Impact on genetic susceptibility and clinical outcome of lung cancer. Biomarkers 22: 165-170, 2017.

11. Damen MS, Agca R, Holewijn S, de Graaf J, Dos Santos JC, van Riel PL, Fransen J, Coenen MJ, Nurmohamed MT, Netea MG, et al: IL-32 promoter SNP rs4786370 predisposes to modified lipoprotein profiles in patients with rheumatoid arthritis. Sci Rep 7: 41629, 2017.

12. Seo EH, Kang J, Kim KH, Cho MC, Lee S, Kim HJ, Kim JH, Kim EJ, Park DK, Kim SH, et al: Detection of expressed IL-32 in human stomach cancer using ELISA and immunostaining. J Microbiol Biotechnol 18: 1606-1612, 2008.

13. Xuan W, Huang W, Wang R, Chen C, Chen Y, Wang Y and Tan X: Elevated circulating IL-32 presents a poor prognostic outcome in patients with heart failure after myocardial infarction. Int $\mathrm{J}$ Cardiol 243: 367-373, 2017.

14. Alehagen U, Ericsson A and Dahlström U: Are there any significant differences between females and males in the management of heart failure? Gender aspects of an elderly population with symptoms associated with heart failure. J Card Fail 15: 501-507, 2009.

15. Yancy CW, Jessup M, Bozkurt B, Butler J, Casey DE Jr, Drazner MH, Fonarow GC, Geraci SA, Horwich T, Januzzi JL, et al: 2013 ACCF/AHA guideline for the management of heart failure: Executive summary: A report of the American college of cardiology foundation/american heart association task force on practice guidelines. Circulation 128: 1810-1852, 2013.

16. Qi H, Shen J and Zhou W: Up-regulation of long non-coding RNA THRIL in coronary heart disease: Prediction for disease risk, correlation with inflammation, coronary artery stenosis, and major adverse cardiovascular events. J Clin Lab Anal 34: e23196, 2020.

17. Ebadi N, Ghafouri-Fard S, Taheri M, Arsang-Jang S and Omrani MD: Expression analysis of inflammatory response-associated genes in coronary artery disease. Arch Physiol Biochem: Jan 8: 1-7, 2020. doi: 10.1080/13813455.2019.1708953

18. Das AA, Chakravarty D, Bhunia D, Ghosh S, Mandal PC, Siddiqui KN and Bandyopadhyay A: Elevated level of circulatory sTLT1 induces inflammation through SYK/MEK/ERK signalling in coronary artery disease. Clin Sci (Lond) 133: 2283-2299, 2019.
19. Kura B, Szeiffova Bacova B, Kalocayova B, Sykora M and Slezak J: Oxidative stress-responsive MicroRNAs in heart injury. Int J Mol Sci 21: 358, 2020

20. Tzoulaki I, Castagné R, Boulangé CL, Karaman I, Chekmeneva E, Evangelou E, Ebbels TMD, Kaluarachchi MR, Chadeau-Hyam M, Mosen D, et al: Serum metabolic signatures of coronary and carotid atherosclerosis and subsequent cardiovascular disease. Eur Heart J 40: 2883-2896, 2019.

21. Matsuzawa Y and Lerman A: Endothelial dysfunction and coronary artery disease: Assessment, prognosis, and treatment. Coron Artery Dis 25: 713-724, 2014.

22. Anderson RD, Petersen JW, Mehta PK, Wei J, Johnson BD, Handberg EM, Kar S, Samuels B, Azarbal B, Kothawade K, et al: Prevalence of coronary endothelial and microvascular dysfunction in women with symptoms of ischemia and no obstructive coronary artery disease is confirmed by a new cohort: The NHLBI-sponsored women's ischemia syndrome evaluation-coronary vascular dysfunction (WISE-CVD). J Interv Cardiol 2019: 7169275, 2019.

23. Damen MSMA, Popa CD, Netea MG, Dinarello CA and Joosten LAB: Interleukin-32 in chronic inflammatory conditions is associated with a higher risk of cardiovascular diseases. Atherosclerosis 264: 83-91, 2017.

24. Nold-Petry CA, Rudloff I, Baumer Y, Ruvo M, Marasco D, Botti P, Farkas L, Cho SX, Zepp JA, Azam T, et al: IL-32 promotes angiogenesis. J Immunol 192: 589-602, 2014.

25. Shamoun L, Kolodziej B, Andersson RE and Dimberg J: Protein expression and genetic variation of IL32 and association with colorectal cancer in Swedish patients. Anticancer Res 38: 321-328, 2018.

(i) $($ This work is licensed under a Creative Commons Attribution-NonCommercial-NoDerivatives 4.0 International (CC BY-NC-ND 4.0) License. 